\title{
Spectroscopy of gold atoms isolated in liquid and solid ${ }^{4} \mathrm{He}$ matrices
}

\author{
P. Moroshkin, ${ }^{\text {a),b) }}$ V. Lebedev, ${ }^{\text {b) }}$ and A. Weis ${ }^{\text {b) }}$ \\ Department of Physics, University of Fribourg, Chemin du Musée 3, 1700 Fribourg, Switzerland
}

\begin{abstract}
We present a systematic experimental study of absorption and fluorescence spectra of Au atoms in bulk liquid and solid helium matrices. The results are compared to the spectra of $\mathrm{Cu}$ atoms studied earlier. We investigate the dependence of the observed spectral lineshapes on the helium pressure. The observed splitting of the $5 d^{10} 6 p-5 d^{9} 6 s^{2}$ transitions of Au in hcp solid He gives evidence for an anisotropic trapping site structure formed either by a non-spherical atomic bubble or a four-vacancy center.
\end{abstract}

\section{INTRODUCTION}

Laser spectroscopy of foreign atoms and molecules embedded in liquid and solid He matrices is a powerful technique for studying the structure and dynamics of quantum fluids and solids. Electronic transitions in the atomic dopants are coupled to elementary matrix excitations (phonons), and the laser excitation of the dopant produces a strongly localized phonon wavepacket with a characteristic spatial and frequency spectrum.

Metal atoms in particular are subject to two different types of excitations. On one hand, optically excited valence electrons strongly couple to matrix phonons and induce large amplitude wavepackets that can be described quasiclassically. Excited inner-shell electrons, on the other hand, are screened from the surrounding $\mathrm{He}$ atoms and interact only weakly with the surrounding matrix. In the latter case, the absorption or emission of a photon by the dopant produces only a small number of matrix phonons. The two types of excitation lead to distinctive spectral features in the laser-induced fluorescence emitted by the isolated species. ${ }^{1}$ Outer shell transitions are strongly broadened and blueshifted with respect to the freeatom transition frequencies and have Gaussian-like lineshapes with a very small asymmetry. Under the same conditions the fluorescence spectrum from inner-shell excitations shows almost no shift and possesses a pronounced substructure, consisting of a very narrow zero-phonon line (ZPL) and a broader and redshifted phonon wing (PW).

High-resolution ZPL and PW spectra were extensively studied in experiments on doped helium nanodroplets (for a review see Refs. 2 and 3). In particular, the measured rovibrational spectra of $\mathrm{SF}_{6}{ }^{4}$ and $\mathrm{OCS}^{5}$ were used to determine the droplets' temperature and to prove that the droplets are superfluid. ZPL and PW structures were also observed in the electronic spectra of glyoxal ${ }^{6}$ and several planar organic molecules, such as tetracene, pentacene, etc. ${ }^{7}$

Most previous experiments on atomic and molecular dopants in bulk liquid and solid He matrices have probed the

\footnotetext{
a) Present address: Low Temperature Physics Laboratory, RIKEN, 2-1 Hirosawa, Wako, Saitama 351-0198, Japan. Eletronic mail: petr.moroshkin@riken.jp.

b)URL: http://physics.unifr.ch/fr/page/56/.
}

spectra of valence electron transitions in alkali and alkalineearth atoms (for a review, see Ref. 8). Inner-shell transitions have been observed in the spectra of the isolated rare-earth atoms Tm and Eu. ${ }^{9,10}$ More recently, we have identified both types of excitations in the spectra of copper and gold atoms in helium matrices. ${ }^{11}$ In the present paper we present a detailed spectroscopic study of Au atoms in superfluid, normal fluid, and solid ${ }^{4} \mathrm{He}$.

\section{EXPERIMENT}

\section{A. Experimental setup}

Measurements were performed in liquid and solid ${ }^{4} \mathrm{He}$ at $1.5-3.7 \mathrm{~K}$, in the pressure range of 1-40 bar using the experimental setup described in detail in Refs. 8 and 12. The helium matrix was doped with gold atoms and molecules by means of laser ablation with the radiation from a pulsed Nd:YAGlaser focused onto a solid gold target installed at the bottom of the sample cell. For the experiments in solid He, the second harmonic ( $\lambda=532 \mathrm{~nm}$ ) of the laser is used with a pulse energy of $50 \mathrm{~mJ}$, pulse length of $5 \mathrm{~ns}$, and a repetition rate of 1-2 Hz. The ablation pulses melt a portion of the solid $\mathrm{He}$ sample above the target. Before starting the spectroscopic experiments we turn off the ablation laser and let the sample cool down and solidify again.

The spectroscopic measurements are performed after ablation is completed. During data taking, cluster-fragmenting pulses of the Nd:YAG laser are fired at a rate of $1 \mathrm{~Hz}$ with a pulse energy of $\sim 10 \mathrm{~mJ}$. The spectroscopy laser and the spectrometer exposure are delayed with respect to these fragmentation pulses by $20-100 \mathrm{~ms}$. This procedure is described in detail in our review paper. $^{8}$ In liquid He, the ablation is continued during the spectroscopic measurements proper. In that case the same light source (2nd or 3rd harmonic of the Nd:YAG laser with a repetition rate of $10 \mathrm{~Hz}$ and other parameters as specified above) is used for ablation and excitation.

The implanted dopants in solid He were excited by the third harmonic $(\lambda=355 \mathrm{~nm})$ of the same pulsed Nd:YAG laser or by a tunable optical parametric oscillator (OPO) pumped by another frequency-tripled Nd:YAG laser. The average power of the excitation laser was kept below $10 \mathrm{~mW}$ in 


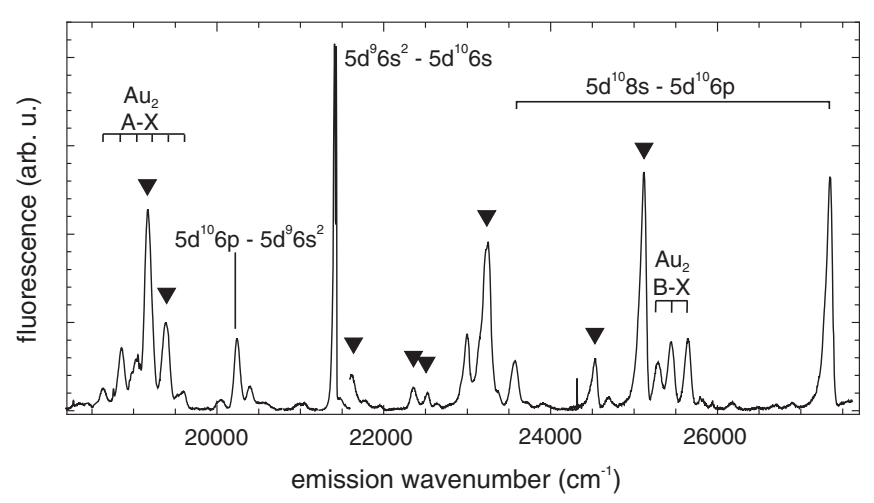

FIG. 1. Laser induced fluorescence spectrum of Au-doped solid ${ }^{4} \mathrm{He}$ at $1.5 \mathrm{~K}$ and 30 bar following excitation by frequency-tripled Nd:YAG radiation at $355 \mathrm{~nm}$. The $5 d^{9} 6 s 7 s-5 d^{9} 6 s 6 p$ transitions are marked by black triangles.

order to prevent melting of the crystal. At a repetition rate of $10 \mathrm{~Hz}$ this corresponds to $1 \mathrm{~mJ} /$ pulse. This laser beam is introduced into the cell via the same path as the ablation laser and focused by the same lens located inside the cryostat, several centimeters above the cell. Care was taken to align the excitation laser beam such that it hits the doped part of the sample. The fluorescence collection optics is aligned such that only light from the observation zone located $\sim 1-2 \mathrm{~cm}$ above the target reaches the spectrometer. The laser beam with a diameter of $\sim 1 \mathrm{~mm}$ crosses this zone in the vertical direction. Therefore atoms ejected from the target by one laser pulse can be excited by subsequent pulses. Fluorescence light from the sample volume was collected by a lens located in the cryostat and then analyzed by a grating spectrograph $(0.1 \mathrm{~nm}$ resolution) equipped with a CCD camera.

\section{B. Fluorescence spectra of valence electron transitions}

Excitation by frequency-tripled Nd:YAG radiation $\left(v_{\text {exc }}\right.$ $=28170 \mathrm{~cm}^{-1}$ ) produces a rich fluorescence spectrum with lines from $\mathrm{Au}$ atoms and vibronic bands from $\mathrm{Au}_{2}$ molecules. Figure 1 shows a typical fluorescence spectrum in the range of $18500-27500 \mathrm{~cm}^{-1}$. In this paper we will focus on the atomic spectra; the spectroscopy of $\mathrm{Au}_{2}$ will be presented in a separate publication. All observed atomic lines and their shifts with respect to the transition frequencies in free $\mathrm{Au}$ atoms are listed in Table I.

In solid He matrices we have identified 12 transitions between highly excited states of the $5 d^{9} 6 s 7 s$ and $5 d^{9} 6 s 6 p$ configurations. Eight of these transitions can be seen in Fig. 1, where they are marked with black triangles. The spectral shifts of these lines with respect to the free atomic lines vary from -140 to $+105 \mathrm{~cm}^{-1}$. In liquid $\mathrm{He}$ we have observed only three transitions from this group, viz., those at 16783,19115 , and $22275 \mathrm{~cm}^{-1}$. These three transitions originate from the $10_{7 / 2}$ level that has the lowest energy in the $5 d^{9} 6 s 7 s$ configuration. ${ }^{13}$ In low pressure liquid He these lines are redshifted with respect to the free-atom lines. With increasing helium pressure the lines broaden and shift towards higher energies at a rate of 2.07(5) $\mathrm{cm}^{-1} /$ bar. Figure 2(a) shows the typical lineshapes of the strongest line of this
TABLE I. Atomic fluorescence lines of Au atoms in solid ${ }^{4} \mathrm{He}(1.5 \mathrm{~K}, 30$ bar) following excitation at $355 \mathrm{~nm}$. The transition energies of the free $\mathrm{Au}$ atom, $v_{\text {free }}$, are taken from Ref. 13.

\begin{tabular}{|c|c|c|c|}
\hline & Transition & $v_{\text {free }}\left(\mathrm{cm}^{-1}\right)$ & Shift $\left(\mathrm{cm}^{-1}\right)$ \\
\hline \multirow{11}{*}{$5 d^{9} 6 s 7 s-5 d^{9} 6 s 6 p$} & $17_{5 / 2}-5_{5 / 2}^{o}$ & 25047 & +72 \\
\hline & $16_{3 / 2}-5_{5 / 2}^{o}$ & 24478 & +80 \\
\hline & $12_{5 / 2}-4_{7 / 2}^{o}$ & 23168 & +77 \\
\hline & $12_{5 / 2}-5_{5 / 2}^{o}$ & 22530 & -7 \\
\hline & $10_{7 / 2}-4_{7 / 2}^{o}$ & 22275 & +81 \\
\hline & $12_{5 / 2}-6_{3 / 2}^{o}$ & 21698 & +65 \\
\hline & $16_{3 / 2}-9_{3 / 2}^{o}$ & 19422 & -46 \\
\hline & $10_{7 / 2}-7_{9 / 2}^{o}$ & 19115 & +27 \\
\hline & $16_{3 / 2}-10_{5 / 2}^{o}$ & 18999 & -142 \\
\hline & $12_{5 / 2}-8_{7 / 2}^{o}$ & 17676 & +105 \\
\hline & $10_{7 / 2}-8_{7 / 2}^{o}$ & 16783 & +95 \\
\hline \multirow{3}{*}{$5 d^{10} 8 s-5 d^{10} 6 p$} & $17_{5 / 2}-16_{3 / 2}^{o}$ & 12377 & -31 \\
\hline & ${ }^{2} S_{1 / 2}-{ }^{2} P_{1 / 2}$ & 27383 & -23 \\
\hline & ${ }^{2} S_{1 / 2}-{ }^{2} P_{3 / 2}$ & 23568 & -27 \\
\hline \multirow[t]{2}{*}{$5 d^{10} 7 s-5 d^{10} 6 p$} & ${ }^{2} S_{1 / 2}-{ }^{2} P_{1 / 2}$ & 17126 & +115 \\
\hline & ${ }^{2} S_{1 / 2}-{ }^{2} P_{3 / 2}$ & 13311 & +99 \\
\hline \multirow[t]{2}{*}{$5 d^{10} 6 p-5 d^{9} 6 s^{2}$} & ${ }^{2} P_{3 / 2}-{ }^{2} D_{3 / 2}$ & 19739 & +500 \\
\hline & ${ }^{2} P_{1 / 2}-{ }^{2} D_{3 / 2}$ & 15924 & +531 \\
\hline $5 d^{9} 6 s^{2}-5 d^{10} 6 s$ & ${ }^{2} D_{3 / 2}-{ }^{2} S_{1 / 2}$ & 21435 & -3 \\
\hline $5 d^{9} 6 s^{2}-5 d^{9} 6 s^{2}$ & ${ }^{2} D_{3 / 2}-{ }^{2} D_{5 / 2}$ & 12274 & +10 \\
\hline
\end{tabular}

group, $10_{7 / 2}-4_{7 / 2}^{o}$, when observed in liquid or in solid He, respectively. The pressure dependence of the lineshifts of the $10_{7 / 2}-4_{7 / 2}^{o}$ and $10_{7 / 2}-7_{9 / 2}^{o}$ transitions is shown in Fig. 2(b).

We have also observed transitions from the $5 d^{10} 8 s$ and $5 d^{10} 7 s$ states to the two $5 d^{10} 6 p^{2} P_{1 / 2,3 / 2}$ states. The $5 d^{10} 8 s-$ $5 d^{10} 6 p$ doublet at $27383 / 23568 \mathrm{~cm}^{-1}$ shown in Fig. 1 could only be observed in solid helium matrices.

We studied the $5 d^{10} 7 s-5 d^{10} 6 p$ doublet at $17126 / 13311$ $\mathrm{cm}^{-1}$ both in liquid and solid He matrices. Typical spectra of the ${ }^{2} S_{1 / 2}-{ }^{2} P_{3 / 2}$ line obtained in liquid and in solid $\mathrm{He}$ and their pressure shift are shown in Figs. 3(a) and 3(b). The line is blueshifted at a rate of $3.00(5) \mathrm{cm}^{-1} /$ bar. The data in Fig. 3(b) suggest that there is an additional increase of the lineshift at the liquid - solid phase transition. Such a
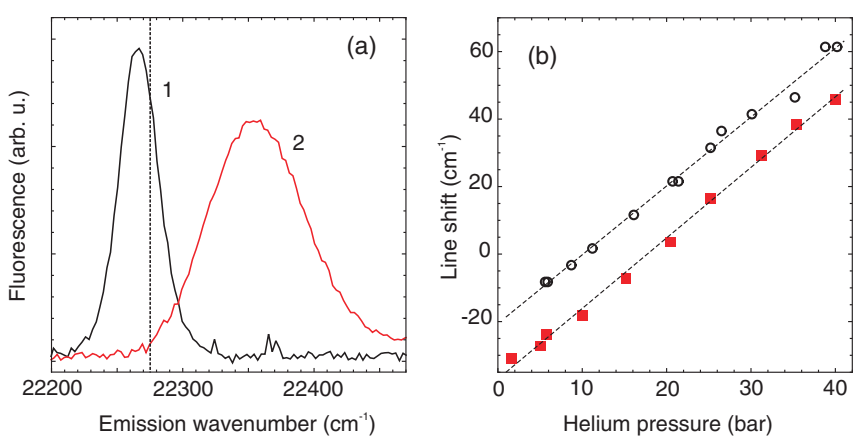

FIG. 2. Fluorescence from $5 d^{9} 6 s 7 s-5 d^{9} 6 s 6 p$ transitions of Au atoms in condensed He following excitation at $355 \mathrm{~nm}$. (a) Lineshapes of the $10_{7 / 2}$ $-4_{7 / 2}^{o}$ line in normal fluid $\mathrm{He}$ at $3.7 \mathrm{~K}$ and 5.6 bar (line 1), and in solid $\mathrm{He}$ at $1.6 \mathrm{~K}$ and $36 \mathrm{bar}$ (line 2). The emission frequency of free Au atoms, $v_{\text {free }}$ $=22275 \mathrm{~cm}^{-1}$, is shown as vertical dashed line. (b) Pressure shift of the $10_{7 / 2}-4_{7 / 2}^{o}$ line (open black circles) and the $10_{7 / 2}-7_{9 / 2}^{o}$ line (red squares) at $T=3.7 \mathrm{~K}$ 

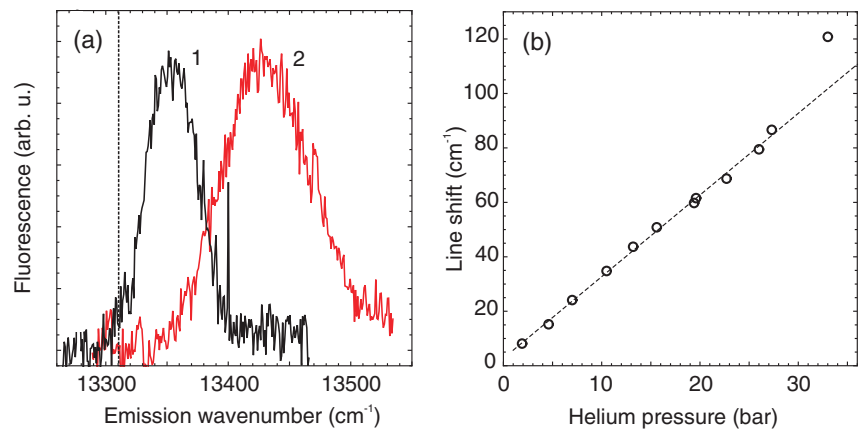

FIG. 3. Fluorescence from $5 d^{10} 7 s-5 d^{10} 6 p$ transitions of Au atoms in condensed He following excitation at $355 \mathrm{~nm}$. (a) Lineshapes of the ${ }^{2} S_{1 / 2}-{ }^{2} P_{3 / 2}$ fluorescence line in superfluid $\mathrm{He}$ at $1.5 \mathrm{~K}$ and 13 bar (line 1), and in solid $\mathrm{He}$ at $1.5 \mathrm{~K}$ and $33 \mathrm{bar}$ (line 2). The emission frequency of free Au atoms, $v_{\text {free }}=13311 \mathrm{~cm}^{-1}$, is shown as vertical dashed line. (b) Pressure shift of the ${ }^{2} S_{1 / 2}-{ }^{2} P_{3 / 2}$ line at $T=1.5 \mathrm{~K}$.

discontinuous increase of the lineshift was observed earlier in the spectra of $\mathrm{Cs}$ and $\mathrm{Cu}$ atoms in condensed $\mathrm{He}^{12,14}$ and was interpreted in the frame of the atomic bubble model. ${ }^{8,14}$ Unfortunately, in the present experiment we were not able to study the lineshift in solid He with a sufficient accuracy since the molecular bands of $\mathrm{Au}_{2}$ dimers dominate this part of the fluorescence spectrum and strongly obscure the atomic line.

The $5 d^{10} 6 p^{2} P_{1 / 2,3 / 2}$ states have two radiative decay channels, viz., transitions towards the $5 d^{10} 6 s^{2} S_{1 / 2}$ ground state and towards the $5 d^{9} 6 s^{2}{ }^{2} D_{3 / 2,5 / 2}$ metastable states, respectively. The strongest lines in the $\mathrm{Au}$ I spectrum of free gold atoms are the $5 d^{10} 6 p^{2} P_{1 / 2,3 / 2}-5 d^{10} 6 s^{2} S_{1 / 2}$ resonance transitions at 37359 and $41175 \mathrm{~cm}^{-1}$ that cannot be detected in our experiment. The same holds for the $5 d^{10} 6{ }^{2} P_{3 / 2}-5 d^{9} 6 s^{2}{ }^{2} D_{5 / 2}$ transition at $32013 \mathrm{~cm}^{-1}$. The two remaining transitions, i.e., $5 d^{10} 6 p^{2} P_{1 / 2,3 / 2}-5 d^{9} 6 s^{2}{ }^{2} D_{3 / 2}$ at 19739 and $15924 \mathrm{~cm}^{-1}$ lie in the visible part of the spectrum and could be detected in our solid He matrix experiments. Both transitions are strongly blueshifted and split into several components. Their typical lineshapes are shown in Fig. 4(a). In contrast to the typical Gaussian-like profiles of other outer-shell transitions, the ${ }^{2} P_{1 / 2}-{ }^{2} D_{3 / 2}$ line has two spectral components that are separated by $\approx 100 \mathrm{~cm}^{-1}$. The ${ }^{2} P_{3 / 2}-{ }^{2} D_{3 / 2}$ line consists of 4
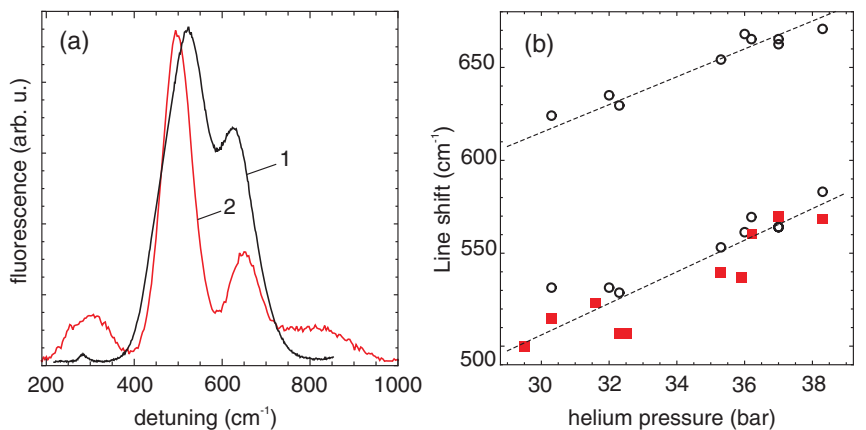

FIG. 4. Fluorescence from $5 d^{10} 6 p-5 d^{9} 6 s^{2}$ transitions of Au atoms in solid He following excitation at $355 \mathrm{~nm}$. (a) Lineshapes of the ${ }^{2} P_{1 / 2,3 / 2}-{ }^{2} D_{3 / 2}$ fluorescence lines at $1.5 \mathrm{~K}$ and 30 bar. Line $1:{ }^{2} P_{1 / 2}-{ }^{2} D_{3 / 2}$ transition $\left(v_{\text {free }}\right.$ $\left.=15924 \mathrm{~cm}^{-1}\right)$; line $2:{ }^{2} P_{3 / 2}-{ }^{2} D_{3 / 2}$ transition $\left(v_{\text {free }}=19739 \mathrm{~cm}^{-1}\right)$. All detunings are relative to the frequency of the free-atomic transition, $v_{\text {free }}$. (b) Pressure shift of the two components of the ${ }^{2} P_{1 / 2}-{ }^{2} D_{3 / 2}$ line (open black circles) and of the main component of the ${ }^{2} P_{3 / 2}-{ }^{2} D_{3 / 2}$ line (red squares).

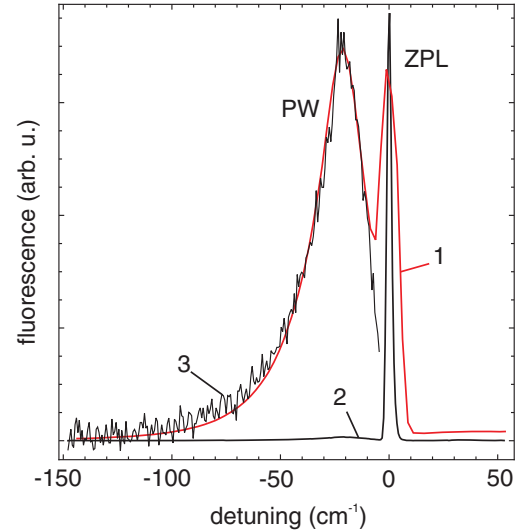

FIG. 5. Emission spectra of the inner-shell transitions of gold atoms in solid $\mathrm{He}$ at $1.5 \mathrm{~K}$ and 32 bar following excitation at $355 \mathrm{~nm}$. Line 1 (red): $5 d^{9} 6 s^{2}$ ${ }^{2} D_{3 / 2}-5 d^{10} 6 s^{2} S_{1 / 2}$ transition $\left(v_{\text {free }}=21435 \mathrm{~cm}^{-1}\right)$; line 2 (black): $5 d^{9} 6 s^{2}$ ${ }^{2} D_{3 / 2}-5 d^{9} 6 s^{2}{ }^{2} D_{5 / 2}$ transition $\left(v_{\text {free }}=12274 \mathrm{~cm}^{-1}\right)$; line 3 : magnified part of line 2 . For each line, zero detuning corresponds to peak of the corresponding narrower zero-phonon line.

components separated by $\approx 160, \approx 150$, and $\approx 190 \mathrm{~cm}^{-1}$. An increase of the helium pressure shifts the components of both transitions to the blue at a rate of $8(1) \mathrm{cm}^{-1} / \mathrm{bar}$, as shown in Fig. 4(b).

\section{Fluorescence spectra of inner-shell transitions}

In solid $\mathrm{He}$, the two forbidden electric quadrupole transitions $5 d^{9} 6 s^{2}{ }^{2} D_{3 / 2}-5 d^{10} 6 s^{2} S_{1 / 2}$ at $21435 \mathrm{~cm}^{-1}$ and $5 d^{9} 6 s^{2}$ ${ }^{2} D_{3 / 2}-5 d^{9} 6 s^{22} D_{5 / 2}$ at $12274 \mathrm{~cm}^{-1}$ happen to be the strongest fluorescence lines emitted by Au atoms. Both lines are almost not shifted with respect to their counterparts in free Au atoms. Their lineshapes, shown in Fig. 5, consist each of two partially overlapping peaks separated by $20 \mathrm{~cm}^{-1}$. The spectral width of the higher energy peak is limited by the resolution of our spectrometer and the lower energy peak is $\approx 30 \mathrm{~cm}^{-1}$ wide.

These lineshapes are very similar to the spectra of the $3 d^{9} 4 s^{2}{ }^{2} D_{3 / 2,5 / 2}-3 d^{10} 4 s^{2} S_{1 / 2}$ transitions of $\mathrm{Cu}$ atoms in solid He matrices reported in Ref. 12. We associate the narrow component with a ZPL and the broader component with a PW. The shift of the zero-phonon component of each line with respect to the corresponding transition in the free atom is very small (see Table I). In order to compare the lineshapes of the two transitions, we represent them in Fig. 5 as a function of the detuning with respect to the corresponding ZPL. The PW of the ${ }^{2} D_{3 / 2}-{ }^{2} D_{5 / 2}$ line is $\approx 50$ times weaker than the PW of the ${ }^{2} D_{3 / 2}-{ }^{2} S_{1 / 2}$ line, while the lineshapes of the two phonon wings are nearly identical.

The spectra of both forbidden lines in solid He matrices do not depend on the helium pressure. In liquid $\mathrm{He}$, the ${ }^{2} D_{3 / 2}$ $-{ }^{2} S_{1 / 2}$ line is much weaker and the ${ }^{2} D_{3 / 2}-{ }^{2} D_{5 / 2}$ line is not visible at all.

In order to determine the decay time of the $5 d^{9} 6 s^{2}{ }^{2} D_{3 / 2}$ state we have tuned the monochromator to $21435 \mathrm{~cm}^{-1}$ and have recorded (with a photodiode) the time-resolved laserinduced fluorescence following pulsed excitation. From this we infer a decay time $\tau$ of 3.0(1) ms, which explains the suppression of the transitions from the $5 d^{9} 6 s^{2}{ }^{2} D_{3 / 2}$ state in 
liquid helium. The metastable gold atoms diffuse out of the observation volume in a time that is shorter than $\tau$ so that their fluorescence does not reach the detector.

\section{D. $5 d^{9} 6 s^{2}-5 d^{10} 6 p$ absorption line}

As discussed in Sec. II C, the excitation of Au-doped solid $\mathrm{He}$ by the frequency-tripled $\mathrm{Nd}$ :YAG laser radiation leads to a very efficient population of the long-lived $5 d^{9} 6 s^{2}$ ${ }^{2} D_{3 / 2}$ state. We have used a tunable OPO to resonantly excite the $5 d^{9} 6 s^{2}{ }^{2} D_{3 / 2}-5 d^{10} 6 p^{2} P_{3 / 2}$ transition $\left(v_{\text {free }}=19739\right.$ $\left.\mathrm{cm}^{-1}\right)$. We observe a strong fine-structure relaxation in the $5 d^{10} 6 p^{2} P_{J}$ states and an efficient population transfer to the $5 d^{10} 6{ }^{2} P_{1 / 2}$ state that can be monitored via fluorescence on the $5 d^{10} 6 p^{2} P_{1 / 2}-5 d^{9} 6 s^{2}{ }^{2} D_{3 / 2}$ transition $\left(v_{\text {free }}=15924\right.$ $\mathrm{cm}^{-1}$ ). In Fig. 6(a) we show the excitation spectrum of the $5 d^{9} 6 s^{2}{ }^{2} D_{3 / 2}-5 d^{10} 6 p{ }^{2} P_{3 / 2}$ line obtained by scanning the OPO frequency in the vicinity of $20000 \mathrm{~cm}^{-1}$ and recording the fluorescence yield on the $5 d^{10} 6 p^{2} P_{1 / 2}-5 d^{9} 6 s^{2}{ }^{2} D_{3 / 2}$ line. The observed excitation line has a particularly large spectral width of $\approx 800 \mathrm{~cm}^{-1}$ and its maximum is blueshifted with respect to the free atomic line by $\approx 400 \mathrm{~cm}^{-1}$. Similarly broadened and shifted absorption lines have been reported for Cs atoms in solid $\mathrm{He}^{15}$ and more recently for $\mathrm{Cr}$ atoms in superfluid He droplets. ${ }^{16}$

\section{DISCUSSION}

\section{A. Observed atomic lines}

The fluorescence spectrum of Au atoms in superfluid $\mathrm{He}$ at saturated vapor pressure has been reported in Ref. 17. In that experiment, highly excited states of $\mathrm{Au}$ were populated via the recombination of $\mathrm{Au}^{+}$ions and free electrons injected into liquid He. The authors of Ref. 17 have observed the recombination-induced fluorescence at the $5 d^{10} 7 s-5 d^{10} 6 p$ fine-structure doublet and the $5 d^{10} 6 p^{2} P_{1 / 2}-5 d^{10} 6 s^{2} S_{1 / 2}$ transition, as well as at some of the $5 d^{9} 6 s 7 s-5 d^{9} 6 s 6 p$ and $5 d^{9} 6 s 6 p-5 d^{9} 6 s^{2}$ transitions. The resonance $5 d^{10} 6 p-5 d^{10} 6 s$ lines and most of the $5 d^{9} 6 s 6 p-5 d^{9} 6 s^{2}$ lines lie in the UV part of spectrum (see Fig. 7) that is not accessible to our experimental setup. The laser-induced Au fluorescence spectrum in liquid He observed in the spectral range of our appara-

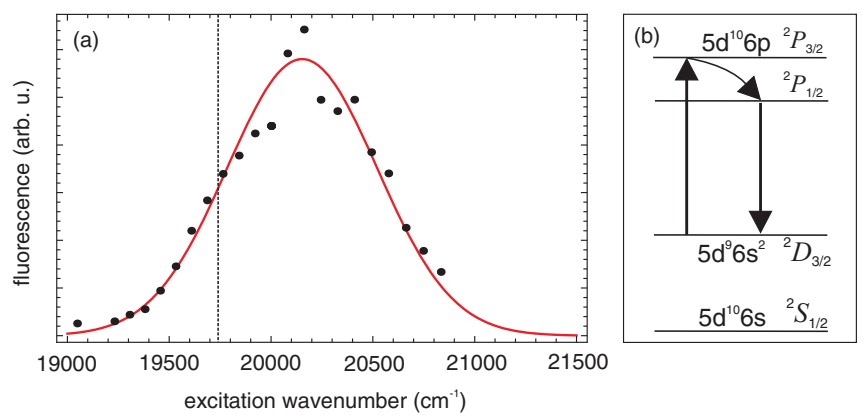

FIG. 6. (a) Excitation spectrum of the $5 d^{9} 6 s^{2}{ }^{2} D_{3 / 2}-5 d^{10} 6 s^{2} P_{3 / 2}$ transition of $\mathrm{Au}$ in solid $\mathrm{He}$ at $1.5 \mathrm{~K}$ and 36 bar. The vertical dashed line marks the frequency of the free atomic transition $\left(v_{\text {free }}=19739 \mathrm{~cm}^{-1}\right)$ and the solid red line is a Gaussian fit to the data. (b) Electronic states of the Au atom involved in the process. tus agrees well with the recombination spectrum of Ref. 17. When extrapolated to saturated vapor pressure, the shifts and widths of the lines observed by us are found to be in good agreement with the values reported in Ref. 17.

The fluorescence spectrum observed in solid He matrices turns out to be much richer than the one in liquid matrices. It can be compared to the laser-induced fluorescence spectrum of $\mathrm{Cu}$ atoms in solid $\mathrm{He}$ studied in our recent work. ${ }^{12}$ In particular, the observed $5 d^{9} 6 s 7 s-5 d^{9} 6 s 6 p$ lines of $\mathrm{Au}$ are much stronger than the corresponding $3 d^{9} 4 s 5 s-3 d^{9} 4 s 4 p$ transitions of $\mathrm{Cu}$, most of which could be detected only in the emission from the ablation spot. The $5 d^{10} 7 s-5 d^{10} 6 p$ and $5 d^{10} 8 s-5 d^{10} 6 p$ fine-structure doublets of gold observed here correspond to the $3 d^{10} 5 s-3 d^{10} 4 p$ and $3 d^{10} 6 s-3 d^{10} 4 p$ doublets of copper studied in Ref. 12. The observed lineshapes and the pressure shifts of the $5 d^{10} 7 s-5 d^{10} 6 p$ lines of $\mathrm{Au}$ (Fig. 3) closely reproduce those of the $3 d^{10} 5 s-3 d^{10} 4 p$ lines of $\mathrm{Cu}$.

We find, however, a pronounced difference between the spectra of $\mathrm{Cu}$ and $\mathrm{Au}$ atoms for transitions originating from the $5 d^{10} 6 p$ states, viz., the $5 d^{10} 6 p^{2} P_{1 / 2,3 / 2}-5 d^{9} 6 s^{2}{ }^{2} D_{3 / 2}$ transitions studied here (Fig. 4) and the $5 d^{10} 6 p^{2} P_{1 / 2}-5 d^{10} 6 s$ ${ }^{2} S_{1 / 2}$ line reported in Ref. 17. In copper, the corresponding transitions originating from the $3 d^{10} 4 p$ states are completely quenched, both in liquid and in solid helium. ${ }^{12,17}$

In Ref. 12 we have suggested a quenching mechanism that involves the formation of $\mathrm{Cu}\left(3 d^{10} 4 p\right) \mathrm{He}_{N}$ quasimolecules (exciplexes) with subsequent radiationless transitions towards the metastable $3 d^{9} 4 s^{2}$ states. Exciplexes are formed when one or several $\mathrm{He}$ atoms penetrate into the nodal region of the metal atom's valence electron cloud. The spin-orbit coupling in the $3 d^{10} 4 p$ state of $\mathrm{Cu}$ (fine structure splitting $\Delta=250$ $\mathrm{cm}^{-1}$ ) is weak compared to the $\mathrm{Cu}-\mathrm{He}$ interaction. In that case, the valence electron density distribution of $\mathrm{Cu}$ can be represented by a dumbbell-shaped spin-orbit uncoupled $p$ orbital, which permits exciplex formation.

The spin-orbit coupling in the $5 d^{10} 6 p$ state of $\mathrm{Au}$, on the other hand, is much stronger $\left(\Delta=3815 \mathrm{~cm}^{-1}\right)$ and the valence electron density is represented by spin-orbit coupled $\left|P_{J}, M_{J}\right\rangle$ orbitals. For the ${ }^{2} P_{1 / 2}$ state the electron density distribution has a repulsive spherical symmetry that prevents exciplex formation.

The above situation closely resembles the well studied formation of exciplex molecules by alkali atoms. ${ }^{18}$ The light alkali atoms $\mathrm{Li}, \mathrm{Na}$, and $\mathrm{K}$ have a weak spin-orbit coupling ( $\Delta=0.3,17$, and $58 \mathrm{~cm}^{-1}$, respectively) and their resonance lines are completely quenched by exciplex formation. On the other hand, the $6^{2} P_{1 / 2}$ state of Cs $\left(\Delta=554 \mathrm{~cm}^{-1}\right)$ is not quenched at all, while the $5^{2} P_{1 / 2}$ state of $\mathrm{Rb}\left(\Delta=238 \mathrm{~cm}^{-1}\right)$ represents an intermediate case: it is not quenched in low pressure liquid $\mathrm{He}$, but becomes quenched in pressurized liquid and in solid He. ${ }^{19}$

The exciplex model outlined above predicts a strong quenching of the ${ }^{2} P_{3 / 2}$ states that have non-spherical orbitals. In agreement with this model, the authors of Ref. 17 did not observe any fluorescence at the $5 d^{10} 6 p{ }^{2} P_{3 / 2}-5 d^{10} 6 s{ }^{2} S_{1 / 2}$ transition of $\mathrm{Au}$ in liquid He. Based on the same argument it is surprising that we observe laser-induced fluorescence at the $5 d^{10} 6 p^{2} P_{3 / 2}-5 d^{9} 6 s^{2}{ }^{2} D_{3 / 2}$ transition. However, the 
observed line is rather weak and can be observed only in solid matrices. As discussed in Sec. II D, laser excitation of the $5 d^{10} 6 p{ }^{2} P_{3 / 2}$ state leads to a strong fluorescence from the lower lying $5 d^{10} 6 p{ }^{2} P_{1 / 2}$ state. We therefore conclude that the $5 d^{10} 6 p^{2} P_{3 / 2}$ state is partially quenched, but that this quenching is less efficient than in the case of copper and alkali-metals.

\section{B. Trapping site structure}

With the exception of the $5 d^{10} 8 s^{2} S_{1 / 2}-5 d^{10} 6 p^{2} P_{1 / 2}$ lineshape that has a pronounced asymmetry, all observed lines from the $5 d^{9} 6 s 7 s-5 d^{9} 6 s 6 p, 5 d^{10} 7 s-5 d^{10} 6 p$, and $5 d^{10} 8 s-$ $5 d^{10} 6 p$ multiplets have symmetric Gaussian-like shapes, similar to the well-studied resonance lines of alkali-metals. ${ }^{14,20}$ Their spectral shifts increase linearly with the helium pressure and are of the same order of magnitude as those of the alkalis. This suggests that the atomic bubble model that successfully predicts the spectra of the alkali-metal atoms in liquid and solid $\mathrm{He}$ is also valid for Au.

Unfortunately, we have not been able to model the fluorescence and excitation spectra studied in the present work, since interaction potentials between excited states of Au atom and ground state $\mathrm{He}$ atoms are not available. Using ground state $\mathrm{Au}-\mathrm{He}$ potentials from Refs. 21 and 22, we have applied the standard atomic bubble model (details are given, e.g., in Ref. 8) to obtain the atomic bubble parameters of the $\mathrm{Au}$

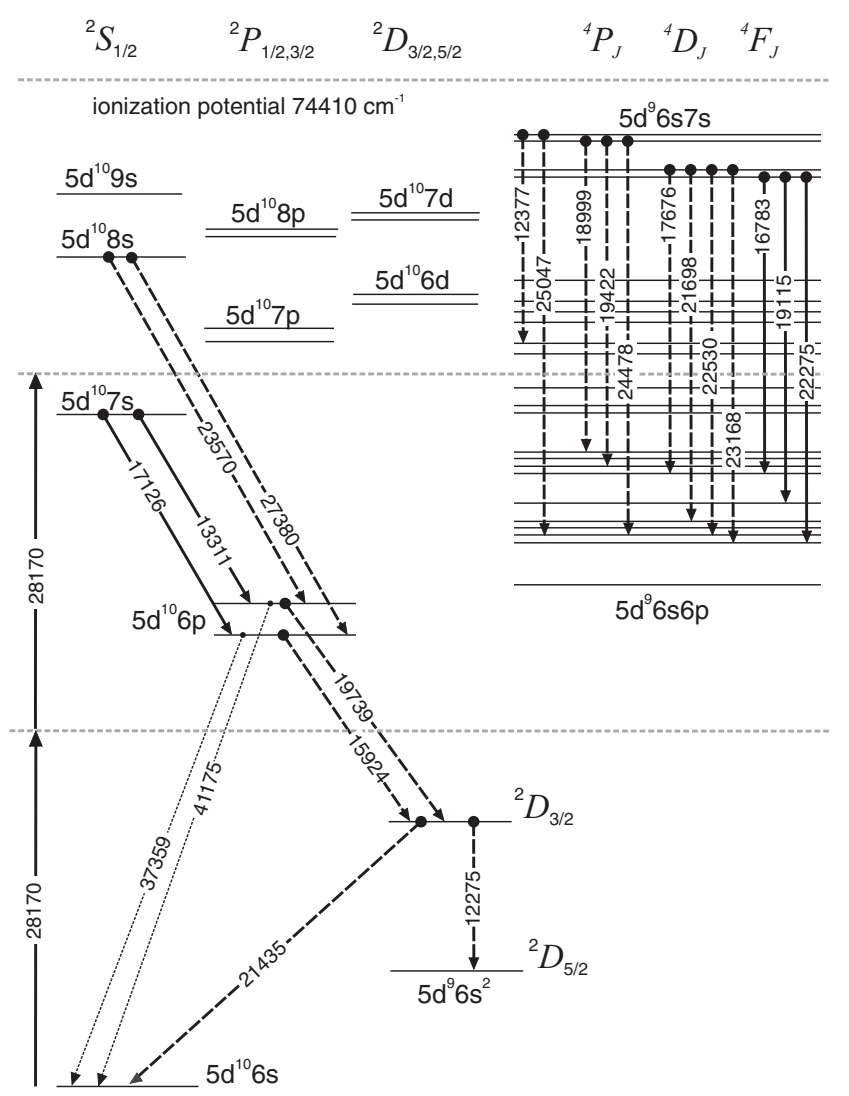

FIG. 7. Electronic states of the Au atom. The down-arrows indicate electronic transitions observed in liquid and solid $\mathrm{He}$ (solid lines) and in solid $\mathrm{He}$ only (dashed lines). The dotted arrows indicate the two resonant transitions that could not be observed. Transition energies are given in $\mathrm{cm}^{-1}$. ground state. The He density around the Au atom, $\rho(\mathbf{R})$, is modeled by the radial trial function

$$
\begin{gathered}
\rho\left(R, R_{0}, \alpha\right)= \\
\left\{\begin{array}{ll}
0, & R<R_{0} \\
\rho_{0}\left[1-\left\{1+\alpha\left(R-R_{0}\right)\right\} e^{-\alpha\left(R-R_{0}\right)}\right], & R \geq R_{0}
\end{array},\right.
\end{gathered}
$$

where $\rho_{0}$ is the density of bulk condensed helium. The total energy of the atom + bubble system is calculated as

$$
\begin{gathered}
E_{\mathrm{tot}}=E_{\mathrm{int}}+p V_{\mathrm{bub}}+\sigma S_{\mathrm{bub}}+ \\
\frac{\hbar^{2}}{8 M_{H e}} \int \frac{(\nabla \rho(R))^{2}}{\rho(R)} d^{3} R+E_{\text {elastic }},
\end{gathered}
$$

where $p$ is the helium pressure, $\sigma$ the surface tension parameter, $V_{\text {bub }}$ and $S_{\text {bub }}$ the volume and surface area of the bubble, $E_{\text {int }}$ the copper-helium interaction energy, and $E_{\text {elastic }}$ the energy due to the stress created in the solid matrix by the inclusion of the bubble. In the liquid phase, $E_{\text {elastic }}=0$, whereas in solid He, $E_{\text {elastic }}=\beta\left(\frac{4 \pi}{3} R_{b u b}^{3}-V_{0}\right)$. Here, $V_{0}$ is the volume of the bubble in liquid He close to the solidification point and $\beta$ is the compressibility of solid helium. ${ }^{15}$ The parameters $R_{0}$ and $\alpha$ were adjusted in order to minimize the total bubble energy given by Eq. (2).

For pressurized liquid helium at $1.5 \mathrm{~K}$ close to the solidification point at 26 bar we obtain $R_{0}=3.2 \AA$ and $\alpha=4.5 \AA^{-1}$. The corresponding bubble radius, $R_{b}$, defined as the center of gravity of the interface profile, is found to be $3.6 \AA$. This is significantly smaller than typical bubble radii of alkali-metal atoms $\left(R_{b} \approx 6 \AA^{14,20}\right)$ and even smaller than the radius of an atomic bubble formed by a copper atom $\left(R_{b}=4 \AA^{12}\right)$ under the same conditions. The Au-He interaction potential ${ }^{21,22}$ has a deeper potential well than the $\mathrm{Cu}-\mathrm{He}$ potential and its repulsive part is located at shorter radial distances. This leads to a smaller bubble radius and a stronger localization of the bubble interface (larger value of $\alpha$ ).

As noted in Ref. 12, such a small atomic bubble fits into the void formed by four adjacent vacancies in the hcp helium crystal. It therefore seems legitimate to propose a fourvacancy center as an alternative trapping site to the (conventional) spherically symmetric atomic bubble structure.

\section{Inner-shell vs. valence electron transitions}

As pointed out in Sec. I, our study was motivated by the possibility to observe both inner-shell and valence electron transitions under the same conditions and to compare their lineshapes. The observed spectra reproduce the general trend reported earlier. ${ }^{1,12}$ Valence electron transitions have very broad structureless lineshapes, whereas the inner-shell transitions possess a characteristic ZPL-PW substructure. The intensity ratio of the ZPL and PW features reflects the strength of the atom-phonon coupling. A stronger coupling leads to higher probability to excite a phonon and therefore results in a higher intensity of the phonon wing in the lineshape. As discussed in Ref. 1, the coupling strength is determined by the magnitude of the change induced in the atomic bubble configuration by the electronic transition in the dopant. We therefore suggest that the ${ }^{2} D_{3 / 2}-{ }^{2} D_{5 / 2}$ transition within the $5 d^{9} 6 s^{2}$ configuration (12 $\left.275 \mathrm{~cm}^{-1}\right)$ induces a minimal change in the bubble shape and size. On the other hand, 
the ${ }^{2} D_{3 / 2}-{ }^{2} S_{1 / 2}$ transition between the $5 d^{9} 6 s^{2}$ and $5 d^{10} 6 s$ configurations $\left(21435 \mathrm{~cm}^{-1}\right)$ induces a stronger displacement of the bubble interface.

As discussed in Ref. 1, the motion of the bubble interface can be represented as a wavepacket of matrix phonons. If the displacement of the interface is not too large, the lineshape of the PW provides information about its spectrum and dynamics. The shapes of the PW features of both transitions shown in Fig. 5 are practically indistinguishable. From this we conclude that the bubble dynamics are essentially the same in both cases, but that they evolve with different amplitudes.

The two $3 d^{9} 4 s^{2}{ }^{2} D_{3 / 2,5 / 2}-3 d^{10} 4 s^{2} S_{1 / 2}$ transitions of $\mathrm{Cu}$ studied in Ref. 12 belong to the same type of transition as the ${ }^{2} D_{3 / 2}-{ }^{2} S_{1 / 2}$ line of Au discussed above. The lineshapes of their PWs are nearly identical to those reported in the present work and the ZPL-PW ratios are close to that of the ${ }^{2} D_{3 / 2}$ $-{ }^{2} S_{1 / 2}$ line. We conclude that all four transitions induce the same kind of motion of the atomic bubble interface, most likely a breathing mode. This is not surprising since the calculated atomic bubble radii for $\mathrm{Au}$ and $\mathrm{Cu}^{12}$ are very similar. We therefore expect the resonant frequencies and the damping constants of the bubble vibrations to be approximately the same.

\section{Splitting of the $5 d^{10} 6 p-5 d^{9} 6 s^{2}$ fluorescence lines}

As discussed in Refs. 8 and 15, the anisotropic stiffness of hep helium crystals leads to a non-spherical shape of the atomic bubble arising due to the $E_{\text {elastic }}$ term in Eq. (2). A four-vacancy center has an even lower symmetry. The interaction with the non-spherical trapping site lifts the degeneracy of the $M_{J}$ sublevels of the electronic states of the Au atom. A state with a half-integer total angular momentum $J$ will split into $J+1 / 2$ doubly degenerate substates with different values of $\left|M_{J}\right|$. As a consequence, transitions between two states with angular momenta $J_{1}$ and $J_{2}$ will split into $3 / 2\left(J_{1}+J_{2}\right)-1 / 2(|\Delta J|+1)$ components with relative intensities determined by the transition dipole matrix elements and the sublevel populations of the initial state.

From the above one therefore expects a doublet structure for the ${ }^{2} S_{1 / 2}-{ }^{2} P_{3 / 2}$ transitions. However, we could not observe such a splitting in the fluorescence spectra of the $5 d^{10} 7 s-5 d^{10} 6 p$ and $5 d^{10} 8 s-5 d^{10} 6 p$ transitions of $\mathrm{Au}$ in the present experiment, and we conclude that any possible splitting of the $5 d^{10} 6 p^{2} P_{3 / 2}$ state in the trapping sites of the $5 d^{10} 7 s$ and $5 d^{10} 8 s^{2} S_{1 / 2}$ states is small compared to the transition linewidth of $\approx 100 \mathrm{~cm}^{-1}$.

On the other hand, the $5 d^{10} 6 p-5 d^{9} 6 s^{2}$ transitions exhibit a very pronounced splitting. In agreement with the above predictions, the ${ }^{2} P_{1 / 2}-{ }^{2} D_{3 / 2}$ line has a double-peak structure, while the ${ }^{2} P_{3 / 2}-{ }^{2} D_{3 / 2}$ line splits into four components. Both lines also have a pressure-dependent blueshift that is much larger than that of the $5 d^{10} 7 s-5 d^{10} 6 p$ and $5 d^{10} 8 s-5 d^{10} 6 p$ transitions. This strong perturbation gives evidence of a strong mismatch between the shapes of the electron density distributions of the initial and final states.

\section{SUMMARY}

In summary, we have carried out a detailed spectroscopic study of $\mathrm{Au}$ atoms isolated in liquid and solid $\mathrm{He}$ matrices. We have observed a number of valence electron transitions as well as transitions of inner-shell electrons. There is a strong resemblance between the spectra of Au atoms studied here and those of $\mathrm{Cu}$ atoms reported earlier. However, the quenching of the $(n-1) d^{10} n p$ states is less pronounced in $\mathrm{Au}$ $(n=6)$ than in $\mathrm{Cu}(n=4)$ under the same conditions. We attribute this effect to the much stronger spin-orbit coupling in the $\mathrm{Au}$ atom.

\section{ACKNOWLEDGMENTS}

This work was supported by Grant No. 200020-119786 of the Schweizerischer Nationalfonds.

${ }^{1}$ P. Moroshkin, V. Lebedev, and A. Weis, Europhys. Lett. 96, 26002 (2011).

${ }^{2}$ J. P. Toennies and A. F. Vilesov, Angew. Chem., Int. Ed. 43, 2622 (2004).

${ }^{3}$ C. Callegari, and W. E. Ernst, in Handbook of High-Resolution Spectroscopy, edited by M. Quack and F. Merkt (Wiley, 2011), Vol. 3, pp. 1551-1594.

${ }^{4}$ M. Hartmann, R. E. Miller, J. P. Toennies, and A. Vilesov, Phys. Rev. Lett. 75, 1566 (1995).

${ }^{5}$ S. Grebenev, J. P. Toennies, and A. F. Vilesov, Science 279, 2083 (1998).

${ }^{6}$ M. Hartmann, F. Mielke, J. P. Toennies, A. F. Vilesov, and G. Benedek, Phys. Rev. Lett. 76, 4560 (1996).

${ }^{7}$ M. Hartmann, A. Lindinger, J. P. Toennies, and A. F. Vilesov, Phys. Chem. Chem. Phys. 4, 4839 (2002).

${ }^{8}$ P. Moroshkin, A. Hofer, and A. Weis, Phys. Rep. 469, 1 (2008).

${ }^{9}$ K. Ishikawa, A. Hatakeyama, K. Gosyono-o, S. Wada, Y. Takahashi, and T. Yabuzaki, Phys. Rev. B 56, 780 (1997)

${ }^{10}$ Q. Hui and M. Takami, J. Low Temp. Phys. 119, 393 (2000).

${ }^{11}$ P. Moroshkin, V. Lebedev, and A. Weis, J. Low Temp. Phys. 162, 710 (2011).

${ }^{12}$ P. Moroshkin, V. Lebedev, and A. Weis, Phys. Rev. A 84, 052519 (2011).

${ }^{13}$ J. C. Ehrhardt and S. P. Davis, J. Opt. Soc. Am. 61, 1342 (1971).

${ }^{14}$ A. Hofer, P. Moroshkin, S. Ulzega, D. Nettels, R. Müller-Siebert, and A. Weis, Phys. Rev. A 76, 022502 (2007).

${ }^{15}$ V. Lebedev, P. Moroshkin, and A. Weis, J. Phys. Chem. A 115, 7169 (2011).

${ }^{16}$ A. Kautsch, M. Koch, and W. E. Ernst, "Electronic relaxation after resonant laser excitation of $\mathrm{Cr}$ in superfluid helium nanodroplets," J. Phys. Chem. A (published online)

${ }^{17}$ B. Tabbert, M. Beau, H. Günther, W. Häu $\beta$ ler, C. Hönninger, K. Meyer, B. Plagemann, and G. zu Putlitz, Z. Phys. B 97, 425 (1995).

${ }^{18}$ J. Dupont-Roc, Z. Phys. B 98, 383 (1995).

${ }^{19}$ T. Kinoshita, K. Fukuda, T. Matsuura, and T. Yabuzaki, Phys. Rev. A 53, 4054 (1996).

${ }^{20}$ T. Kinoshita, K. Fukuda, Y. Takahashi, and T. Yabuzaki, Phys. Rev. A 52, 2707 (1995).

${ }^{21}$ F. Cargnoni, T. Kus, M. Mella, and R. J. Bartlett, J. Chem. Phys. 129, 204307 (2008).

${ }^{22}$ X. F. Tong, C. L. Yang, Y. P. An, M. S. Wang, X. G. Ma, and D. H. Wang, J. Chem. Phys. 131, 244304 (2009). 